Research Article

\title{
Certain Class of Analytic Functions Connected with $q$-Analogue of the Bessel Function
}

\author{
Nazek Alessa $\mathbb{D}^{1},{ }^{1}$ B. Venkateswarlu, ${ }^{2}$ K. Loganathan $\left(\mathbb{D},{ }^{3}\right.$ T.S. Karthik, ${ }^{4}$ \\ P. Thirupathi Reddy, ${ }^{5}$ and G. Sujatha ${ }^{2}$ \\ ${ }^{1}$ Department of Mathematical Sciences, Faculty of Science, Princess Nourah Bint Abdulrahman University, Riyadh, Saudi Arabia \\ ${ }^{2}$ Department of Mathematics, GSS, GITAM University, Bengaluru Rural, Doddaballapur 562 163, Karnataka, India \\ ${ }^{3}$ Research and Development Wing, Live4Research, Tiruppur 638 106, Tamilnadu, India \\ ${ }^{4}$ Department of Electronics and Communication Engineering, Aditya College of Engineering and Technology, \\ Surampalem 533 437, Andhra Pradesh, India \\ ${ }^{5}$ Department of Mathematics, Kakatiya University, Warangal 506 009, Telangana, India
}

Correspondence should be addressed to K. Loganathan; loganathankaruppusamy304@gmail.com

Received 20 February 2021; Revised 19 March 2021; Accepted 27 March 2021; Published 13 April 2021

Academic Editor: Ming-Sheng Liu

Copyright (c) 2021 Nazek Alessa et al. This is an open access article distributed under the Creative Commons Attribution License, which permits unrestricted use, distribution, and reproduction in any medium, provided the original work is properly cited.

The focus of this article is the introduction of a new subclass of analytic functions involving $q$-analogue of the Bessel function and obtained coefficient inequities, growth and distortion properties, radii of close-to-convexity, and starlikeness, as well as convex linear combination. Furthermore, we discussed partial sums, convolution, and neighborhood properties for this defined class.

\section{Introduction}

Let $A$ specify the category of analytic functions and $\eta$ represent on the unit disc $\Delta=\{w:|w|<1\}$ with normalization $\eta(0)=0$ and $\eta^{\prime}(0)=1$ such that a function has the extension of the Taylor series on the origin in the form

$$
\eta(w)=w+\sum_{\nu=2}^{\infty} a_{\nu} w^{\nu}
$$

Indicated by $S$, the subclass of $A$ is composed of functions that are univalent in $\Delta$.

Then, a $\eta(w)$ function of $A$ is known as starlike and convex of order $\mathcal{Y}$ if it delights the pursing

$$
\begin{array}{r}
\mathscr{R}\left\{\frac{w \eta^{\prime}(w)}{\eta(w)}\right\}>\vartheta, \quad w \in \Delta, \\
\mathscr{R}\left\{1+\frac{w \eta^{\prime \prime}(w)}{\eta^{\prime}(w)}\right\}>\vartheta, \quad w \in \Delta,
\end{array}
$$

for specific $\vartheta(0 \leq \vartheta<1)$, respectively, and we express by $S^{*}(\vartheta)$ and $K(\vartheta)$ the subclass of $A$, which is expressed by the aforesaid functions, respectively. Also, indicated by $T$, the subclass of $A$ is made up of functions of this form

$$
\eta(w)=w-\sum_{\nu=2}^{\infty} a_{\nu} w^{\nu}, \quad a_{\nu} \geq 0, w \in \Delta,
$$

and let $T^{*}(\vartheta)=T \cap S^{*}(\vartheta), C(\vartheta)=T \cap K(\vartheta)$. There are interesting properties in the $T^{*}(\vartheta)$ and $C(\vartheta)$ classes which were thoroughly studied by Silverman [1] and Alessa et al. [2].

The intense devotion of scientists has recently fascinated the study of the $q$-calculus. The great focus in many fields of mathematics and physics is due to its benefits. In the analysis of many subclasses of analytic functions, the importance of the $q$-derivative operator $D_{q}$ is very evident from its applications.

The concept of $q$-star functions was originally proposed by Ismail et al. [3] in the year 1990. However, in the sense of 
Geometric Function Theory, a firm basis of the use of the $q$-calculus was effectively developed. For example, in the rotational flow of Burge's fluid flowing through an unbounded round channel [3], it is used to derive velocity and stress.

After that, numerous mathematicians have carried out remarkable studies, which play a significant role in the development of geometric function theory. Furthermore, Srivastava [4] recently published a survey-cum-expository analysis article that could be useful for researchers and scholars working on these topics. The mathematical description and applications of the fractional $q$-calculus and fractional $q$-derivative operators in geometric function theory were systematically investigated in this surveycum-expository analysis article [4]. In particular, Srivastava et al. [5] also considered some function groups of conical region related $q$-star like functions. For other recent investigations involving the $q$-calculus, one may refer to [6-13].

One of the most significant special functions is the Bessel function. As a result, it is important for solving a wide range of problems in engineering, physics, and mathematics (see [14]). In recent years, several researchers have focused their efforts on forming different types of relationships. Many researchers have recently focused on determining the various conditions under which a Bessel function has geometric properties such as close-to-convexity, starlikeness, and convexity in the frame of a unit disc $\Delta$.

The first-order Bessel function $\wp$ is defined by the infinite series [15]:

$$
\mathscr{F}_{\wp}(w):=\sum_{\nu=0}^{\infty} \frac{(-1)^{\nu}(w / 2)^{2 \nu+\wp}}{\nu ! \Gamma(\nu+\wp+1)}, \quad w \in \mathbb{C}, \wp \in \mathbb{R},
$$

where $\Gamma$ stands for a function of Gamma. Szasz and Kupan [16] and Thirupathi Reddy and Venkateswarlu [17] have recently explored the univalence of the first-kind normalization Bessel function $k_{\wp}: \Delta \longrightarrow \mathbb{C}$ defined by

$$
\begin{aligned}
k_{\wp}(w) & :=2^{\wp} \Gamma(\wp+1) w^{1-(\wp / 2)} \mathcal{F}_{\wp}\left(w^{(1 / 2)}\right) \\
& =w+\sum_{\nu=2}^{\infty} \frac{(-1)^{\nu-1} \Gamma(\wp+1)}{4^{\nu-1}(\nu-1) ! \Gamma(\nu+\wp)} w^{\nu}, \quad w \in \Delta, \wp \in \mathbb{R} .
\end{aligned}
$$

For $0<q<1$, El-Deeb and Bulboaca [18] defined the $q$-derivative $k_{\wp}$ operator as follows:

$$
\begin{aligned}
\partial_{q} k_{\wp}(w) & =\partial_{q}\left(w+\sum_{\nu=2}^{\infty} \frac{(-1)^{\nu}-1 \Gamma(\wp+1)}{4^{\nu-1}(\nu-1) ! \Gamma(\nu+\wp)} w^{\nu}\right):=\frac{k_{\wp}(q w)-k_{\wp}(w)}{w(q-1)} \\
& =1+\sum_{\nu=2}^{\infty} \frac{(-1)^{\nu-1} \Gamma(\wp+1)}{4^{\nu-1}(\nu-1) ! \Gamma(\nu+\wp)}[\nu, q] w^{\nu-1}, \quad w \in \Delta,
\end{aligned}
$$

where

$$
[\nu, q]:=\frac{1-q^{\nu}}{1-q}=1+\sum_{j=1}^{\nu-1} q^{j}, \quad[0, q]:=0 .
$$

Using (7), we are going to define two products in the text:

(1) The $q$-shifted factorial is given for any nonnegative integer $v$ :

$$
[v, q]:= \begin{cases}1, & \text { if } \nu=0 \\ {[1, q][2, q], \ldots,[k, q],} & \text { if } \nu \in \mathbb{N}\end{cases}
$$

(2) The $q$-generalized Pochhammer symbol for any positive number $r$ is defined by

$$
[r, q]_{v}:= \begin{cases}1, & \text { if } \nu=0, \\ [r, q][r+1, q], \ldots,[r+k-1,]], & \text { if } \nu \in \mathbb{N} .\end{cases}
$$

For $\wp>0, \ell>-1$, and $0<q<1$, El-Deeb and Bulboaca [18] defined $\mathcal{J}_{\wp, q}^{\ell}: \Delta \longrightarrow \mathbb{C}$ by (see [19])

$$
\mathscr{J}_{\wp, q}^{\ell}(w):=w+\sum_{\nu=2}^{\infty} \frac{(-1)^{\nu-1} \Gamma(\wp+1)}{4^{\nu-1}(\nu-1) ! \Gamma(\nu+\wp)} \frac{[\nu, q] !}{[\ell+1, q]_{\nu-1}} w^{\nu}
$$

A simple computation shows that

$$
\mathscr{J}_{\wp, q}^{\ell}(w) * \mathscr{M}_{q, \ell+1}(w)=w \partial_{q} k_{\wp}(w), \quad w \in \Delta,
$$

where the function $\mathscr{M}_{q, \ell+1}(w)$ is supplied with the function

$$
\mathscr{M}_{q, \ell+1}(w):=w+\sum_{\nu=2}^{\infty} \frac{[\ell+1, q]_{v-1}}{[\nu-1, q] !} w^{\nu}, \quad w \in \Delta .
$$

El-Deeb and Bulboaca [18] introduced the linear operator using the definition of $q$-derivative along with the idea of convolutions $\mathscr{N}_{\mathfrak{\wp}, q}^{\ell}: \mathscr{A} \longrightarrow \mathscr{A}$ defined by 


$$
\begin{aligned}
& \mathcal{N}_{\wp, q}^{\ell} \eta(w):=\mathscr{J}_{\wp, q}^{\ell}(w) * \eta(w) \\
& =w+\sum_{\nu=2}^{\infty} \Upsilon_{\nu, q}(\wp, \ell) a_{\nu} w^{\nu}, \quad \wp>0, \ell>-1,0<q<1, w \in \Delta, \\
& \text { where } \Upsilon_{\nu, q}(\wp, \ell):=\frac{(-1)^{\nu-1} \Gamma(\wp+1)}{4^{\nu-1}(\nu-1) ! \Gamma(\nu+\wp)} \frac{[\nu, q] !}{[\ell+1, q]_{\nu-1}} \text {. }
\end{aligned}
$$

Remark 1 (see [18]). We can easily verify from the definition relation (13) that the next relation holds for all $\eta \in \mathscr{A}$ :

$$
\begin{aligned}
& {[\ell+1, q] \mathcal{N}_{\wp, q}^{\ell} \eta(w)=[\ell, q] \mathcal{N}_{\wp, q}^{\ell+1} \eta(w)+q^{\ell} w \partial_{q}\left([\ell+1, q] \mathcal{N}_{\wp, q}^{\ell+1} \eta(w)\right), \quad w \in \Delta} \\
& \lim _{q \longrightarrow 1^{-}} \mathcal{N}_{\wp, q}^{\ell} \eta(w)=\mathscr{J}_{\wp, 1}^{\ell} \eta(w):=\mathscr{J}_{\wp}^{\ell} \eta(w) \\
& =w+\sum_{\nu=2}^{\infty} \frac{(-1)^{\nu-1} \Gamma(\wp+1)}{4^{\nu-1}(\nu-1) ! \Gamma(\nu+\wp)} \frac{\nu !}{(\ell+1)_{\nu-1}} a_{\nu} w^{\nu}, \quad w \in \Delta . \\
& \sum_{\nu=2}^{\infty}[\nu+\hbar \nu(\nu-1)-\vartheta] \Upsilon_{\nu, q}(\wp, \ell)\left|a_{\nu}\right| \leq 1-\vartheta
\end{aligned}
$$

Now, we propose a new subclass $\phi_{\wp, q}^{\ell}(\hbar, \vartheta)$ of $A$ concerning $q$ - analogue of the Bessel function as follows.

Definition 1. For $0 \leq \hbar<1,0 \leq \vartheta<1, \wp>0, \ell>-1$ and $0<q<1$, we say $\eta(w) \in A$ is in $\phi_{\wp, q}^{\ell}(\hbar, \vartheta)$ if it fulfils the requirement

$$
\mathscr{R}\left(\frac{w\left(\mathscr{N}_{\wp, q}^{\ell} \eta(w)\right)^{\prime}+\hbar w^{2}\left(\mathcal{N}_{\wp, q}^{\ell} \eta(w)\right)^{\prime \prime}}{\mathcal{N}_{\wp, q}^{\ell} \eta(w)}\right)>\vartheta, \quad w \in \Delta .
$$

Also, we indicate by $T \phi_{\wp, q}^{\ell}(\hbar, \vartheta)=\phi_{\wp, q}^{\ell}(\hbar, \vartheta) \cap T$.

\section{Coefficient Inequalities}

This section gives us an adequate requirement for a function $\eta$ given by $(1)$ to be in $\phi_{\wp, q}^{\ell}(\hbar, \vartheta)$.

Theorem 1. A function $\eta \in A$ is assigned to the class $\phi_{\wp, q}^{\ell}(\hbar, \vartheta)$ if
Proof. Since $0 \leq \vartheta<1$ and $\hbar \geq 0$, now if we put

$$
\varrho(w)=\frac{w\left(\mathcal{N}_{\wp, q}^{\ell} \eta(w)\right)^{\prime}+\hbar w^{2}\left(\mathcal{N}_{\wp, q}^{\ell} \eta(w)\right)^{\prime \prime}}{\mathcal{N}_{\wp, q}^{\ell} \eta(w)}, \quad w \in \Delta,
$$

rhen its just a matter of proving it $|\varrho(w)-1|<1-\vartheta, w \in \Delta$. Indeed, if $\eta(w)=w(w \in \Delta)$, then we have $\varrho(w)=w(w \in \Delta)$.

This implies (16) holds.

If $\eta(w) \neq w(|w|=r<1)$, then there exist a coefficient $\Omega_{\nu}(\wp, \ell) a_{\nu} \neq 0$ for some $\nu \geq 2$. The consequence is that $\sum_{\nu=2}^{\infty} \Upsilon_{\nu, q}(\wp, \ell)\left|a_{\nu}\right|>0$. Further note that

$$
\begin{aligned}
\sum_{\nu=2}^{\infty}[\nu+\hbar \nu(\nu-1)-\vartheta] \Upsilon_{\nu, q}(\wp, \ell)\left|a_{\nu}\right| & >(1-\vartheta) \sum_{\nu=2}^{\infty} \Upsilon_{\nu, q}(\wp, \ell)\left|a_{\nu}\right| \\
& \Longrightarrow \sum_{\nu=2}^{\infty} \Upsilon_{\nu, q}(\wp, \ell)\left|a_{\nu}\right|<1
\end{aligned}
$$


By (16), we obtain

$$
\begin{aligned}
{[\varrho(w)-1] } & =\left|\frac{\sum_{\nu=2}^{\infty}[\nu+\hbar \nu(\nu-1)-1] \Upsilon_{\nu, q}(\wp, \ell) a_{\nu} w^{\nu-1}}{1+\sum_{\nu=2}^{\infty} \Upsilon_{\nu, q}(\wp, \ell) a_{\nu} w^{\nu-1}}\right| \\
& <\frac{\sum_{\nu=2}^{\infty}[\nu+\hbar \nu(\nu-1)-1] \Upsilon_{\nu, q}(\wp, \ell)\left|a_{\nu}\right|}{1-\sum_{\nu=2}^{\infty} \Upsilon_{\nu, q}(\wp, \ell)\left|a_{\nu}\right|} \\
& \leq \frac{\sum_{\nu=2}^{\infty}[\nu+\hbar \nu(\nu-1)-\vartheta] \Upsilon_{\nu, q}(\wp, \ell)\left|a_{\nu}\right|-(1-\vartheta) \Upsilon_{\nu, q}(\wp, \ell)\left|a_{\nu}\right|}{1-\sum_{\nu=2}^{\infty} \Upsilon_{\nu, q}(\wp, \ell)\left|a_{\nu}\right|} \\
& \leq \frac{(1-\vartheta)-(1-\vartheta) \sum_{\nu=2}^{\infty} \Upsilon_{\nu, q}(\wp, \ell)\left|a_{\nu}\right|}{1-\sum_{\nu=2}^{\infty} \Upsilon_{\nu, q}(\wp, \ell)\left|a_{\nu}\right|} \\
& =1-\vartheta, \quad w \in \Delta .
\end{aligned}
$$

Hence, we obtain

$$
\mathscr{R}\left(\frac{w\left(\mathcal{N}_{\wp, q}^{\ell} \eta(w)\right)^{\prime}+\hbar w^{2}\left(\mathcal{N}_{\wp, q}^{\ell} \eta(w)\right)^{\prime \prime}}{\mathcal{N}_{\wp, q}^{\ell} \eta(w)}\right)=\mathscr{R}(\varrho(w))>1-(1-\vartheta)=\vartheta .
$$

Then, $\eta \in \phi_{\wp, q}^{\ell}(\hbar, \vartheta)$.

Theorem 2. Let $\eta$ be given by (3). Then, the function

$$
\eta \in T \phi_{\wp, q}^{\ell}(\hbar, \vartheta) \Longleftrightarrow \sum_{\nu=2}^{\infty}[\nu+\hbar \nu(\nu-1)-\vartheta] \Upsilon_{\nu, q}(\wp, \ell)\left|a_{\nu}\right| \leq 1-\vartheta .
$$

Proof. In view of Theorem 1 , to examine it, $\eta \in T \phi_{\wp, q}^{\ell}(\hbar, \vartheta)$ fulfils the coefficient inequality (16). If $\eta \in T \phi_{\wp, q}^{\ell}(\hbar, \vartheta)$, then the function

$$
\varrho(w)=\frac{w\left(\mathcal{N}_{\wp, q}^{\ell} \eta(w)\right)^{\prime}+\hbar w^{2}\left(\mathcal{N}_{\wp, q}^{\ell} \eta(w)\right)^{\prime \prime}}{\mathcal{N}_{\wp, q}^{\ell} \eta(w)}, \quad w \in \Delta,
$$

satisfies $\mathscr{R}(\varrho(w))>\vartheta$. This implies that

$$
\mathcal{N}_{\wp, q}^{\ell} \eta(w)=w-\sum_{\nu=2}^{\infty} \Upsilon_{\nu, q}(\wp, \ell)\left|a_{\nu}\right| w^{\nu} \neq 0, \quad w \in \Delta \backslash\{0\} .
$$

Noting that $\left(\mathcal{N}_{\wp, q}^{\ell} \eta(r) / r\right)$ in the open interval $(0,1)$, this is the real continuous function with $\eta(0)=1$, and we have

$$
\frac{\mathcal{N}_{\wp, q}^{\ell} \eta(r)}{r}=1-\sum_{\nu=2}^{\infty} \Upsilon_{\nu, q}(\wp, \ell)\left|a_{\nu}\right| r^{\nu-1}>0, \quad 0<r<1
$$

Now,

$$
\vartheta<\varrho(r)=\frac{1-\sum_{\nu=2}^{\infty}[\nu+\hbar \nu(\nu-1)] \Upsilon_{\nu, q}(\wp, \ell)\left|a_{\nu}\right| r^{\nu-1}}{1-\sum_{\nu=2}^{\infty} \Upsilon_{\nu, q}(\wp, \ell)\left|a_{\nu}\right| r^{\nu-1}}
$$

and consequently, by (24), we obtain

$$
\sum_{\nu=2}^{\infty}[\nu+\hbar \nu(\nu-1)-\vartheta] \Upsilon_{\nu, q}(\wp, \ell)\left|a_{\nu}\right| r^{\nu-1} \leq 1-\vartheta .
$$

Letting $r \longrightarrow 1$, we get $\sum_{\nu=2}^{\infty}[\nu+\hbar \nu(\nu-1)-\vartheta] \Upsilon_{\nu, q}$ $(\wp, \ell)\left|a_{\nu}\right| \leq 1-\vartheta$.

This proves the converse part.

Remark 2. If a function $\eta$ of form (3) belongs to the class $T \phi_{\wp, q}^{\ell}(\hbar, \vartheta)$, then

$$
\left|a_{\nu}\right| \leq \frac{1-\vartheta}{[\nu+\hbar \nu(\nu-1)-\vartheta] \Upsilon_{\nu, q}(\wp, \ell)}, \quad \nu \geq 2 .
$$

The equality holds for the functions

$$
\eta_{\nu}(w)=w-\frac{1-\vartheta}{[\nu+\hbar \nu(\nu-1)-\vartheta] \Upsilon_{\nu, q}(\wp, \ell)} w^{\nu}, \quad w \in \Delta, \nu \geq 2
$$




\section{Distortion Theorem}

Theorem 3. Let $\eta \in T \phi_{\wp, q}^{\ell}(\hbar, \vartheta)$ and $|w|=r<1$. Then,

In the section, the distortion limits of the functions are owned by the class $T \phi_{\wp, q}^{\ell}(\hbar, \vartheta)$.

$$
\begin{gathered}
r-\frac{1-\vartheta}{[2 \hbar-\vartheta+2] \Upsilon_{2, q}(\wp, \ell)} r^{2} \leq|\eta(w)| \leq r+\frac{1-\vartheta}{[2 \hbar-\vartheta+2] \Upsilon_{2, q}(\wp, \ell)} r^{2}, \\
1-\frac{2(1-\vartheta)}{[2 \hbar-\vartheta+2] \Upsilon_{2, q}(\wp, \ell)} r \leq\left|\eta^{\prime}(w)\right| \leq 1+\frac{2(1-\vartheta)}{[2 \hbar-\vartheta+2] \Upsilon_{2, q}(\wp, \ell)} r .
\end{gathered}
$$

The approximation is sharp, with the $\eta_{2}(w)$ extreme function indicated by (28).
Proof. Since $\eta \in T \phi_{\wp, q}^{\ell}(\hbar, \vartheta)$, we apply Theorem 2 to attain

$$
\begin{array}{r}
{[2 \hbar-\vartheta+2] \Upsilon_{2, q}(\wp, \ell) \sum_{\nu=2}^{\infty}\left|a_{\nu}\right| \leq \sum_{\nu=2}^{\infty}[\nu+\hbar \nu(\nu-1)-\vartheta] \Upsilon_{\nu, q}(\wp, \ell)\left|a_{\nu}\right| \leq 1-\vartheta} \\
\text { Thus, }|\eta(w)| \leq|w|+|w|^{2} \sum_{\nu=2}^{\infty}\left|a_{\nu}\right| \leq r+\frac{1-\vartheta}{[2 \hbar-\vartheta+2] \Upsilon_{2, q}(\wp, \ell)} r^{2}
\end{array}
$$

$$
\text { Also, we have }|\eta(w)| \leq|w|-|w|^{2} \sum_{\nu=2}^{\infty}\left|a_{\nu}\right| \leq r-\frac{1-\vartheta}{[2 \hbar-\vartheta+2] \Upsilon_{2, q}(\wp, \ell)} r^{2}
$$

Equation (29) follows. In a similar way, for $\eta^{\prime}$, the inequalities

$$
\begin{aligned}
& \left|\eta^{\prime}(w)\right| \leq 1+\sum_{\nu=2}^{\infty} v\left|a_{\nu}\right||w|^{\nu-1} \leq 1+|w| \sum_{\nu=2}^{\infty} v\left|a_{\nu}\right|, \\
& \sum_{\nu=2}^{\infty} v\left|a_{\nu}\right| \leq \frac{2(1-\vartheta)}{[2 \hbar-\vartheta+2] \Upsilon_{2, q}(\wp, \ell)}
\end{aligned}
$$

are satisfied, which leads to (30).

\section{Radii of Close-to-Convexity and Starlikeness}

A close-to-convex and star-like radius of this class $T \phi_{\wp, q}^{\ell}(\hbar, \vartheta)$ is obtained in this section.

Theorem 4. Let $\eta$ be specified by (3) in $T \phi_{\wp, q}^{\ell}(\hbar, \vartheta)$. Then, $\eta$ is the order of close-to-convex $\ell(0 \leq \ell<1)$ in the disc $|w|<t_{1}$, where

$$
t_{1}=\inf _{\nu \geq 2}\left[\frac{(1-\ell)[\nu+\nu \hbar(\nu-1)-\vartheta] \Upsilon_{\nu, q}(\wp, \ell)}{\nu(1-\vartheta)}\right]^{1 /(\nu-1)} .
$$

The estimate is sharp with the extremal function $\eta(w)$ is indicated by (28).

Proof. If $\eta \in T$ and $\eta$ is order of close-to-convex $\ell$, then we obtain

$$
\left|\eta^{\prime}(w)-1\right| \leq 1-\ell
$$

For the L.H.S of (34), we obtain

$$
\left|\eta^{\prime}(w)-1\right| \leq \sum_{\nu=2}^{\infty} v a_{\nu}|w|^{\nu-1}<1-\ell \Rightarrow \sum_{\nu=2}^{\infty} \frac{\nu}{1-\ell} a_{\nu}|w|^{\nu-1} \leq 1 .
$$

We know that

$$
\begin{aligned}
\eta(w) \in T \phi_{\wp, q}^{\ell}(\hbar, \vartheta) \Longleftrightarrow & \sum_{\nu=2}^{\infty} \frac{[\nu+\nu \hbar(\nu-1)-\vartheta] \Upsilon_{\nu, q}(\wp, \ell)}{(1-\vartheta)} \\
& \cdot a_{\nu} \leq 1 .
\end{aligned}
$$

Thus, (34) holds true if 


$$
\begin{aligned}
\frac{\nu}{1-\ell}|w|^{\nu-1} & \leq \frac{[\nu+\nu \hbar(\nu-1)-\vartheta] \Upsilon_{\nu, q}(\wp, \ell)}{(1-\vartheta)}, \\
& \Longrightarrow|w| \leq\left[\frac{(1-\ell)[\nu+\nu \hbar(\nu-1)-\vartheta] \Upsilon_{\nu, q}(\wp, \ell)}{\nu(1-\vartheta)}\right]^{1 /(\nu-1)},
\end{aligned}
$$

and hence proved.

Theorem 5. Let $\eta \in T \phi_{\wp, q}^{\ell}(\hbar, \vartheta)$. Then, $\eta$ is order of starlike $\ell(0 \leq \ell<1)$ in the disc $|w|<t_{2}$, where

$$
t_{2}=\inf _{\nu \geq 2}\left[\frac{(1-\ell)[\nu+\nu \hbar(\nu-1)-\vartheta] \Upsilon_{\nu, q}(\wp, \ell)}{(\nu-\ell)(1-\vartheta)}\right]^{1 /(\nu-1)} .
$$

The estimate is sharp with the extremal function $\eta(w)$ indicated by (28).

Proof. We have $\eta \in T$ and $\eta$ is order of starlike $\ell$, and we have

$$
\left|\frac{w \eta^{\prime}(w)}{\eta(w)}-1\right|<1-\ell
$$

For the L.H.S of (39), we have

$$
\left|\frac{w \eta^{\prime}(w)}{\eta(w)}-1\right| \leq \frac{\sum_{\nu=2}^{\infty}(\nu-1) a_{\nu}|w|^{\nu-1}}{1-\sum_{\nu=2}^{\infty} a_{\nu}|w|^{\nu-1}} .
$$

$(1-\ell)$ is bigger than the R.H.S of the left relation if

$$
\sum_{\nu=2}^{\infty} \frac{v-\ell}{1-\ell} a_{\nu}|w|^{\nu-1}<1 .
$$

We know that

$$
\eta \in T \phi_{\wp, q}^{\ell}(\hbar, \vartheta) \Longleftrightarrow \sum_{\nu=2}^{\infty} \frac{[\nu+\nu \hbar(\nu-1)-\vartheta] \Upsilon_{\nu, q}(\wp, \ell)}{(1-\vartheta)} a_{\nu} \leq 1 .
$$

Thus, (39) is true if

$$
\begin{aligned}
\frac{\nu-\ell}{1-\ell}\|w\|^{\nu-1} & \leq \frac{[\nu+\nu \hbar(\nu-1)-\vartheta] \Upsilon_{\nu, q}(\wp, \ell)}{(1-\vartheta)} \\
& \Longrightarrow|w| \leq\left[\frac{(1-\ell)[\nu+\nu \hbar(\nu-1)-\vartheta] \Upsilon_{\nu, q}(\wp, \ell)}{(\nu-\ell)(1-\vartheta)}\right]^{1 /(\nu-1)} .
\end{aligned}
$$

It yields starlikeness of the family.

\section{Convex Linear Combinations}

Theorem 6. Let $\eta_{1}(w)=w$ and

$$
\eta_{\nu}(w)=w-\frac{1-\vartheta}{[\nu+\hbar \nu(\nu-1)-\vartheta] \Upsilon_{\nu, q}(\wp, \ell)} w^{\nu}, \quad w \in \Delta, \nu \geq 2
$$

Then, $\eta \in T \phi_{\wp, q}^{\ell}(\hbar, \vartheta) \Longleftrightarrow \eta$ in the way it can be expressed:

$$
\eta(w)=\sum_{\nu=1}^{\infty} \mu_{\nu} \eta_{\nu}(w), \quad \mu_{\nu} \geq 0
$$

and $\sum_{\nu=1}^{\infty} \mu_{\nu}=1$.

Proof. If a function $\eta$ is of the form $\eta(w)=\sum_{\nu=1}^{\infty}$ $\mu_{\nu} \eta_{\nu}(w), \mu_{v} \geq 0$ and $\sum_{v=1}^{\infty} \mu_{v}=1$, then

$$
\sum_{\nu=2}^{\infty}[\nu+\hbar \nu(\nu-1)-\vartheta] \Upsilon_{\nu, q}(\wp, \ell)\left|a_{\nu}\right|
$$

$$
\begin{aligned}
& =\sum_{\nu=2}^{\infty}[\nu+\hbar \nu(\nu-1)-\vartheta] \Upsilon_{\nu, q}(\wp, \ell) \\
& \cdot \frac{(1-\vartheta) \mu_{v}}{[\nu+\hbar \nu(\nu-1)-\vartheta] \Upsilon_{\nu, q}(\wp, \ell)} \\
& =\sum_{\nu=2}^{\infty}(1-\vartheta) \mu_{\nu}=\left(1-\mu_{1}\right)(1-\vartheta) \\
& \leq(1-\vartheta),
\end{aligned}
$$

which provides (21), and hence $\eta \in T \phi_{\wp, q}^{\ell}(\hbar, \vartheta)$, by Theorem 2 .

On the contrary, if $\eta$ is in the class $\eta \in T \phi_{\wp, q}^{\ell}(\hbar, \vartheta)$, then we may set 


$$
\mu_{\nu}=\frac{[\nu+\hbar \nu(\nu-1)-\vartheta] \Upsilon_{\nu, q}(\wp, \ell)}{1-\vartheta}\left|a_{\nu}\right|, \quad \nu \geq 2,
$$

and $\mu_{1}=1-\sum_{\nu=2}^{\infty} \mu_{\nu}$.

Then, the function $\eta$ is of form (45).

\section{Partial Sums}

Silverman [20] examined partial sums $\eta$ for the function $\eta \in A$ given by (1) and established through

$$
\begin{aligned}
\eta_{1}(w) & =w \\
\eta_{m}(w) & =w+\sum_{\nu=2}^{m} a_{\nu} w^{\nu}, \quad m=2,3,4, \ldots
\end{aligned}
$$

In this paragraph, in the class $\phi_{\wp, q}^{\ell}(\hbar, \vartheta)$, partial function sums can be considered and sharp lower limits can be reached for the function. True component ratios are $\eta$ to $\eta_{m}$ and $\eta^{\prime}$ to $\eta_{m}^{\prime}$.

Theorem 7. Let $\eta \in \phi_{\wp, q}^{\ell}(\hbar, \vartheta)$ fulfil (16). Then,

$$
\mathscr{R}\left(\frac{\eta(w)}{\eta_{m}(w)}\right) \geq 1-\frac{1}{d_{m+1}}, \quad w \in \Delta, m \in \mathbb{N},
$$

where

$$
d_{\nu}=\frac{[\nu+\hbar \nu(\nu-1)-\vartheta]}{1-\vartheta}
$$

Proof. Clearly, $d_{\nu+1}>d_{v}>1, v=2,3,4, \ldots$

Thus, by Theorem 1, we obtain

$$
\sum_{\nu=2}^{\infty}\left|a_{\nu}\right|+d_{m+1} \sum_{\nu=2}^{\infty}\left|a_{\nu}\right| \leq \sum_{\nu=2}^{\infty} d_{\nu}\left|a_{\nu}\right| \leq 1 .
$$

$$
\begin{gathered}
\text { Setting } g(w)=d_{m+1}\left\{\frac{\eta(w)}{\eta_{m}(w)}-\left(1-\frac{1}{d_{m+1}}\right)\right\}, \\
g(w)=1+\frac{d_{m+1} \sum_{\nu=m+1}^{\infty} a_{\nu} w^{\nu-1}}{1+\sum_{\nu=2}^{m} a_{\nu} w^{\nu-1}},
\end{gathered}
$$

and it is good enough to show $\mathscr{R}(g(w))>0, w \in \Delta$. Applying (51), we think that

$$
\begin{array}{r}
\left|\frac{g(w)-1}{g(w)+1}\right| \leq \frac{d_{m+1} \sum_{\nu=2}^{\infty}\left|a_{\nu}\right|}{2-2 \sum_{\nu=2}^{m}\left|a_{\nu}\right|-d_{m+1} \sum_{\nu=m+1}^{\infty}\left|a_{\nu}\right|} \\
\leq 1,
\end{array}
$$

which gives

$$
\mathscr{R}\left(\frac{\eta(w)}{\eta_{m}(w)}\right) \geq 1-\frac{1}{d_{m+1}},
$$

and hence proved.

Theorem 8. Let $\eta$ in $T \phi_{\wp, q}^{\ell}(\hbar, \vartheta)$ fulfil (16). Then,

$$
\mathscr{R}\left(\frac{\eta_{m}(w)}{\eta(w)}\right) \geq \frac{d_{m+1}}{1+d_{m+1}}, \quad w \in \Delta, m \in \mathbb{N},
$$

where

$$
d_{\nu}=\frac{[\nu+\hbar \nu(\nu-1)-\vartheta]}{1-\vartheta}
$$

Proof. Clearly, $d_{\nu+1}>d_{v}>1, v=2,3,4, \ldots$

Thus, by Theorem 1, we obtain

$$
\begin{aligned}
\sum_{\nu=2}^{\infty}\left|a_{\nu}\right|+d_{m+1} \sum_{\nu=m+1}^{\infty}\left|a_{\nu}\right| & \leq \sum_{\nu=2}^{\infty} d_{\nu}\left|a_{\nu}\right| \leq 1, \\
\text { Setting } h(w) & =\left(1+d_{m+1}\right)\left\{\frac{\eta_{m}(w)}{\eta(w)}-\left(\frac{d_{m+1}}{1+d_{m+1}}\right)\right\}, \\
h(w) & =1-\frac{\left(1+d_{m+1}\right) \sum_{\nu=m+1}^{\infty} a_{\nu} w^{\nu-1}}{1+\sum_{\nu=2}^{m} a_{\nu} w^{\nu-1}},
\end{aligned}
$$

to show $\mathscr{R}(h(w))>0, w \in \Delta$. Implementing (57), we attain

$$
\begin{aligned}
\left|\frac{h(w)-1}{h(w)+1}\right| & \leq \frac{\left(1+d_{m+1}\right) \sum_{\nu=2}^{\infty}\left|a_{\nu}\right|}{2-2 \sum_{\nu=2}^{\infty}\left|a_{\nu}\right|-\left(1+d_{m+1}\right) \sum_{\nu=m+1}^{\infty}\left|a_{\nu}\right|} \\
& \leq 1,
\end{aligned}
$$

which gives

$$
\mathscr{R}\left(\frac{\eta_{m}(w)}{\eta(w)}\right) \geq \frac{d_{m+1}}{1+d_{m+1}},
$$

and hence proved.

Theorem 9. Let $\eta$ in $T \phi_{\wp, q}^{\ell}(\hbar, \vartheta)$ fulfil (16). Then,

$$
\mathscr{R}\left(\frac{\eta^{\prime}(w)}{\eta_{m}^{\prime}(w)}\right) \geq 1-\frac{m+1}{d_{m+1}}, \quad w \in \Delta, m \in \mathbb{N},
$$

$$
\mathscr{R}\left(\frac{\eta_{m}^{\prime}(w)}{\eta^{\prime}(w)}\right) \geq \frac{d_{m+1}}{m+1+d_{m+1}}, \quad w \in \Delta, m \in \mathbb{N},
$$


where

$$
d_{\nu}=\frac{[\nu+\hbar \nu(\nu-1)-\vartheta]}{1-\vartheta}
$$

Proof. By setting

$$
\begin{aligned}
& g(w)=d_{m+1}\left\{\frac{\eta^{\prime}(w)}{\eta_{m}^{\prime}(w)}-\left(1-\frac{m+1}{d_{m+1}}\right)\right\}, \quad w \in \Delta, \\
& h(w)=\left(m+1+d_{m+1}\right)\left\{\frac{\eta_{m}^{\prime}(w)}{\eta^{\prime}(w)}-\left(\frac{d_{m+1}}{m+1+d_{m+1}}\right)\right\}, \quad w \in \Delta,
\end{aligned}
$$

the evidence is close to that of (51) and (52) theorems, so the specifics are omitted.

\section{Convolution Properties}

We will prove in this section that the $T \phi_{\wp, q}^{\ell}(\hbar, \vartheta)$ class is closed by convolution.

Theorem 10. Let $g(w)$ of the form,

$$
g(w)=w-\sum_{\nu=2}^{\infty} b_{\nu} w^{\nu}
$$

be regular in $\Delta$. If $\eta \in T \phi_{\wp, q}^{\ell}(\hbar, \vartheta)$, then the function $\eta^{*} g$ is in the class $T \phi_{\wp, q}^{\ell}(\hbar, \vartheta)$. Here, the symbol * denotes to the Hadamard product.

Proof. Since $\eta \in T \phi_{\wp, q}^{\ell}(\hbar, \vartheta)$, we have

$$
\sum_{\nu=2}^{\infty}[\nu+\hbar \nu(\nu-1)-\vartheta] \Upsilon_{\nu, q}(\wp, \ell)\left|a_{\nu}\right| \leq 1-\vartheta
$$

Employing the last inequality and the fact that

$$
\left(\eta^{*} g\right)(w)=w-\sum_{\nu=2}^{\infty} a_{\nu} b_{\nu} w^{\nu}
$$

we obtain

$$
\begin{aligned}
& \sum_{\nu=2}^{\infty}[\nu+\hbar \nu(\nu-1)-\vartheta] \Upsilon_{\nu, q}(\wp, \ell)\left|a_{\nu}\right|\left|b_{\nu}\right| \\
& \leq \sum_{\nu=2}[\nu+\hbar \nu(\nu-1)-\vartheta] \Upsilon_{\nu, q}(\wp, \ell)\left|a_{\nu}\right| \\
& \quad \leq 1-\vartheta,
\end{aligned}
$$

and hence, in view of Theorem 1, the result follows.

\section{Neighborhood Property}

Following [21, 22], we defined the $\alpha$-neighbourhood of the function $\eta(w) \in T$ by

$$
\aleph_{\alpha}(\eta)=\left\{g \in T: g(w)=w-\sum_{\nu=2}^{\infty} b_{\nu} w^{\nu} \text { and } \sum_{\nu=2}^{\infty} \nu\left|a_{\nu}-b_{\nu}\right| \leq \alpha\right\} .
$$

Definition 2. The function $\eta \in A$ is defined in the class $T \phi_{\wp, q}^{\ell}(\hbar, \vartheta)$ if the function $h \in T \phi_{\wp, q}^{\ell}(\hbar, \vartheta)$ occurs in such a way that the function is $h \in T \phi_{\wp, q}^{\ell}(\hbar, \vartheta)$ :

$$
\left|\frac{\eta(w)}{h(w)}-1\right|<1-\gamma, \quad w \in \Delta, 0 \leq \gamma<1 .
$$

Theorem 11. If $h \in T \phi_{\wp, q}^{\ell}(\hbar, \vartheta)$ and

$$
\gamma=1-\frac{\alpha(2 \hbar-\vartheta+2) \Upsilon_{2, q}(\wp, \ell)}{(2 \hbar-\vartheta+2) \Upsilon_{2, q}(\wp, \ell)-(1+\vartheta)^{\prime}},
$$

then $\aleph_{\alpha}(h) \subseteq T \phi_{\wp, q}^{\ell, \gamma}(\hbar, \vartheta)$.

Proof. Let $\eta \in \aleph_{\alpha}(h)$. We then find from

$$
\sum_{\nu=2}^{\infty} \nu\left|a_{\nu}-b_{\nu}\right| \leq \alpha,
$$

which easily implies the coefficient inequality

$$
\sum_{\nu=2}^{\infty}\left|a_{\nu}-b_{\nu}\right| \leq \frac{\alpha}{v}
$$

Since $h \in T \phi_{\wp, q}^{\ell}(\hbar, \vartheta)$, we have from equation (16) that

$$
\sum_{\nu=2}^{\infty}\left|a_{\nu}\right| \leq \frac{1-\vartheta}{(2 \hbar-\vartheta+2) \Upsilon_{2, q}(\wp, \ell)},
$$

$$
\begin{aligned}
\left|\frac{\eta(w)}{h(w)}-1\right| & <\frac{\sum_{\nu=2}^{\infty} \nu\left|a_{\nu}-b_{\nu}\right|}{1-\sum_{\nu=2}^{\infty} b_{\nu}} \\
& \leq \frac{\alpha}{2} \frac{(2 \hbar-\vartheta+2) \Upsilon_{2, q}(\wp, \ell)}{(2 \hbar-\vartheta+2) \Upsilon_{2, q}(\wp, \ell)-(1+\vartheta)} \\
& =1-\gamma,
\end{aligned}
$$

and hence proved.

\section{Conclusions}

This research has introduced $q$-analogue of the Bessel function and studied some basic properties of geometric function theory. Accordingly, some results related to coefficient estimates, growth and distortion properties, convex linear combination, partial sums, radii of close-to-convexity 
and starlikeness, convolution, and neighborhood properties have also been considered, inviting future research for this field of study.

\section{Data Availability}

No data were used to support the findings of the study.

\section{Conflicts of Interest}

The authors declare that they have no conflicts of interest.

\section{Authors' Contributions}

All authors contributed equally to this work. And, all the authors have read and approved the final version manuscript.

\section{Acknowledgments}

This research was funded by the Deanship of Scientific Research at Princess Nourah Bint Abdulrahman University through the Fast-track Research Funding Program.

\section{References}

[1] H. Silverman, "Univalent functions with negative coefficients," Proceedings of the American Mathematical Society, vol. 51, no. 1, pp. 109-116, 1975.

[2] N. Alessa, B. Venkateswarlu, P. Thirupathi Reddy, K. Loganathan, and K. Tamilvanan, "A New subclass of analytic functions related to mittag-leffler type poisson distribution series," Journal of Function Spaces, vol. 2021, Article ID 6618163, 7 pages, 2021.

[3] M. E. H. Ismail, E. Merkes, and D. Styer, "A generalization of starlike functions," Complex Variables, Theory and Application: An International Journal, vol. 14, no. 1-4, pp. 77-84, 1990.

[4] H. M. Srivastava, "Operators of basic (or q-) calculus and fractional q-calculus and their applications in geometric function theory of complex analysis," Iranian Journal of Science and Technology, Transactions A: Science, vol. 44, no. 1, pp. 327-344, 2020.

[5] H. M. Srivastava, B. Khan, N. Khan, Q. Z. Ahmad, and M. Tahir, "A generalized conic domain and its applications to certain subclasses of analytic functions," Rocky Mountain Journal of Mathematics, vol. 49, pp. 2325-2346, 2019.

[6] H. Aldweby and M. Darus, "Some subordination results on $q$ analogue of Ruscheweyh differential operator," Abstract and Applied Analysis, vol. 2014, Article ID 958563, 6 pages, 2014.

[7] M. Arif, O. Barkub, H. M. Srivastava, S. Abdullah, and S. A. Khan, "Some Janowski type harmonic q-starlike functions associated with symmetrical points," Mathematics, vol. 8, p. 629, 2020.

[8] Q. Khan, M. Arif, M. Raza, G. Srivastava, and H. Tang, "Some applications of a new integral operator in q-analog for multivalent functions," Mathematics, vol. 7, pp. 1-13, 2019.

[9] B. Khan, Z. G. Liu, H. M. Srivastava, N. Khan, M. Darus, and M. Tahir, "A study of some families of multivalent q-starlike functions involving higher-order q-derivatives," Mathematics, vol. 8, pp. 1-12, 2020.

[10] S. Mahmood, N. Raza, E. S. AbuJarad, G. Srivastava, H. M. Srivastava, and S. N. Malik, "Geometric properties of certain classes of analytic functions associated with a q-integral operator," Symmetry, vol. 11, pp. 1-14, 2019.

[11] T. Janani, G. Murugusundaramoorthy, C. Selvaraj, and O. S. Babu, "Subclasses of starlike functions associated with fractional q-calculus operators," Journal of Complex Analysis, vol. 2013, Article ID 572718, 8 pages, 2013.

[12] M. S. Rehman, Q. Z. Ahmad, H. M. Srivastava, B. Khan, and N. Khan, "Partial sums of generalized q-mittag-leffer functions," AIMS Mathematics, vol. 5, pp. 408-420, 2019.

[13] H. M. Srivastava, M. Tahir, B. Khan, Q. Ahmad, and N. Khan, "Some general families of q-starlike functions associated with the Janowski functions," Filomat, vol. 33, no. 9, pp. 2613-2626, 2019.

[14] S. Hussain, S. Khan, M. A. Zaighum, and M. Darus, "Applications of a q-salagean type operator on multivalent function," Journal of Inequalities and Applications, vol. 2018, pp. 301-312, 2018.

[15] A. Baricz, Generalized Bessel Functions of The First Kind, Springer-Verlag Berlin Heidelberg, Heidelberg, Germany, 1994.

[16] R. Szasz and P. A. Kupan, "About the univalence of the Bessel functions," Bolyai Society Mathematical Studies, vol. 54, pp. 127-132, 2009

[17] P. Thirupathi Reddy and B. Venkateswarlu, "A certain subclass of uniformly convex functions defined by Bessel functions," Proyecciones (Antofagasta), vol. 38, no. 4, pp. 719-731, 2019.

[18] S. M. El-Deeb and T. Bulboaca, "Fekete-szegö ineqalities for certain class of analytic functions connected with $q$-analouge of Bessel function," Journal of the Egyptian Mathematical Society, vol. 27, no. 1, p. 42, 2019.

[19] S. M. El-Deeb, "Maclaurin coefficient estimates for new subclasses of bi-univalent functions connected with a q-analogue of bessel function," Abstract and Applied Analysis, vol. 2020, Article ID 8368951, 7 pages, 2020.

[20] H. Silverman, "Partial sums of starlike and convex functions," Journal of Mathematical Analysis and Applications, vol. 209, no. 1, pp. 221-227, 1997.

[21] A. W. Goodman, "Univalent functions and nonanalytic curves," Proceedings of the American Mathematical Society, vol. 8, no. 3, pp. 598-601, 1957.

[22] S. Ruscheweyh, "Neighborhoods of univalent functions," Proceedings of the American Mathematical Society, vol. 81, no. 4, pp. 521-527, 1981. 